\title{
Film Edge Nonlocal Spin Valves
}

\author{
Andrew T. McCallum and Mark Johnson \\ Naval Research Laboratory, Washington, DC 20375, USA*
}

\begin{abstract}
Spintronics is a new paradigm for integrated digital electronics. Recently established as a niche for nonvolatile magnetic random access memory (MRAM), it offers new functionality while demonstrating low power and high speed performance. However, to reach high density spintronic technology must make a transition to the nanometer scale. Prototype devices are presently made using a planar geometry and have an area determined by the lithographic feature size, currently about $100 \mathrm{~nm}$. Here we present a new nonplanar geometry in which one lateral dimension is given by a film thickness, the order of $10 \mathrm{~nm}$. With this new approach, cell sizes can shrink by an order of magnitude. The geometry is demonstrated with a nonlocal spin valve, where we study devices with an injector/detector separation much less than the spin diffusion length.
\end{abstract}

Spintronics is a unique approach to information processing that utilizes the spin state of a conduction electron, rather than its charge ${ }^{1}$. The dominant device families include two sandwich structures, the giant magnetoresistance (GMR) spin valve and the magnetic tunnel junction (MTJ), and the lateral spin valve which is described below. All devices have operational similarity. Their structure includes two single domain ferromagnetic layers, F1 and F2, that are separated by a nonmagnetic layer, N. The magnetization M1 of F1 is typically "pinned," constrained to lie in a given direction. Layer F2, called the free layer, is created in a way that its magnetization, M2, has a uniaxial anisotropy such that M2 can be oriented parallel or antiparallel with M1. GMR spin valves and MTJs are characterized by a low (high) electric resistance when M1 and M2 are parallel (antiparallel). The difference between resistive values of the parallel and antiparallel configurations, relative to the former, is the magnetoresistance ratio (MR) that characterizes the device. The N layer of an MTJ is a thin dielectric tunnel barrier, and that of a GMR spin valve is a thin layer of nonmagnetic metal such as copper. For a lateral spin valve, the $\mathrm{N}$ layer is a thin nonmagnetic metal wire. However, the electric resistance is low (high) when M1 and M2 are antiparallel (parallel). 
All these different devices are fabricated using standard top down lithographic processing. Prototype spin valves and MTJs are fabricated as thin film layered stacks with elliptical shape and dimensions of roughly $70 \mathrm{~nm}$ by $130 \mathrm{~nm}$, and have shown excellent characteristics. However, problems with magnetic anisotropies that may arise when dimensions shrink below these values are one reason why prototypes with smaller areal dimensions have found less success for digital electronics applications. Recently, a spin valve with diameter of $40 \mathrm{~nm}$ had a successful demonstration as a microwave resonator ${ }^{2}$.

Nonlocal lateral spin valves (NLSVs) were first fabricated in 1985를. Recently, numerous groups have created devices using many different kinds of metals and semiconductors $, \underline{4}, \underline{6}, \underline{7}, \underline{8}$. Lateral spin valves have been fabricated with dimensions of order $100 \mathrm{~nm}$ and have been used to resolve basic issues of spin transport, such as the spatial separation of spin and charge currents in a nonlocal geometry $\underline{4}^{\underline{4}}$. They can be used to study fundamental properties such as electron spin flip scattering rates and spin polarization properties of ferromagnet / nonmagnet $(\mathrm{F} / \mathrm{N})$ interfaces, properties that are relevant to all spintronic devices $\underline{9} \underline{10}, 11$. Furthermore, the fact that the resistance changes in NLSVs increase with decreasing device size may make these devices practical for future MRAM or sensor applications.

Previous NLSVs have had the same planar geometry, shown in the inset of Fig. 1. Two or more ferromagnetic (FM) electrodes are deposited on top of or under a narrow nonmagnetic channel $(\mathrm{N})$. These devices are often referred to as lateral spin valves because the FM layers are arranged across the surface of the substrate and not on top of one another as in a GMR structure. When a current $I$ is driven from one of the ferromagnetic electrodes, the injector (F1), a spin accumulation forms in the channel near F1. If the other ferromagnetic electrode, the detector (F2), is close enough to F1 a voltage difference will form across the $\mathrm{F} 2 / \mathrm{N}$ interface in response to the spin accumulation. While this voltage difference can not be measured directly, a voltage $V$ can be measured between F2 and a region of the channel far from the current path, and $V$ includes the voltage difference across the F2/N interface. To isolate the effect of the spin accumulation we measure the nonlocal resistance $R_{N L} \equiv V / I$ with the magnetizations M1 and M2 parallel and antiparallel to one another. The nonlocal resistance change $\Delta R \equiv R_{N L}$ (Parallel)- $R_{N L}$ (Antiparallel) is due to the effect of spin accumulation and is insensitive to the Hall and anisotropic magnetoresistance effects.

The nonlocal resistance change of many NLSVs is proportional to $e^{-L / \delta_{s}}$, where $L$ is the center to center distance between F1 and F2 and $\delta_{s}$ is the spin diffusion length $\underline{3}, 7.12$. This 
exponential dependence is caused by spin flip scattering of electrons in the channel. For a lateral spin valve $L$ must be greater than the minimum (half pitch) lithographic feature size $f$. Since $f$ is the same order of magnitude as $\delta_{s}$ in many materials, $e^{-L / \delta_{s}}$ is much less than 1 and $\Delta R$ is significantly limited.

In this Letter we introduce a nonplanar geometry for NLSVs where the injector/detector spacing $L$ is much less than $\delta_{s}$ and much smaller than in lateral spin valves. By fabricating the device across the edge of a multilayered film stack, the critical dimension (the spacing $L$ ) is determined by the thickness of one of the layers in the stack, the order of $10 \mathrm{~nm}$. The area of our NLSV device, given approximately by the product of $L$ and wire width $w$, is an order of magnitude smaller than that of state of the art planar NLSVs $\mathbf{4} .7$.

These film edge nonlocal (FENL) spin valve devices were fabricated using the edge of a lithographically patterned multilayer film, formed by ion milling with a photoresist mask (Fig. 11). Creating the edge by a removal process allowed the individual layers of the multilayer to terminate cleanly against the edge. The multilayer film consisted of two FM layers that act as F1 and F2, separated and capped with insulating layers. Electrical contact was made separately to each FM layer. Then a narrow metal wire, that acts as the channel, was deposited across the edge of the multilayer film. An examination of the geometry reveals that the center to center spacing between the FM layers can be expressed as $L=$ $\left(\bar{t}_{F M}+t_{I}\right) / \cos (\theta)$, where $\bar{t}_{F M}$ is the average thickness of the two FM layers, $t_{I}$ is the thickness of the insulating layer between the FM layers and $\theta$ is the angle the edge makes from the z-axis. While the geometry of these FENL spin valves is very different from planar lateral spin valves, the fundamental physics describing the flow of electrons through the devices is the same. These FENL spin valves do not include any tunnel barriers and are different from "edge junctions" previously described $\underline{13}$.

The materials used in the devices and their thicknesses were chosen for their electrical and magnetic properties. Starting at the bottom, the multilayer consisted of $10 \mathrm{~nm}$ of $\mathrm{Ni}_{81} \mathrm{Fe}_{19}, 27 \mathrm{~nm}$ of SiN, $10 \mathrm{~nm}$ of $\mathrm{Cog}_{90} \mathrm{Fe}_{10}$, and $27 \mathrm{~nm}$ of SiN on top (refer to Fig. (1). The FM and SiN layers were sputter deposited at a pressure of $2.5 \mathrm{mTorr}$ and $15 \mathrm{mTorr}$ respectively. The FM layers were thick enough to have low coercivities yet thin enough to minimize $L$. The SiN layers were thick enough so that there were no pinholes between the $\mathrm{NiFe}$ and CoFe layers. This was verified by measuring the resistance between these two layers before the $\mathrm{Cu}$ wire was deposited. In our devices M1 is not pinned. However, the 
NiFe and CoFe layers have different coercivities so that sweeping a magnetic field in the ydirection can change the magnetizations of the two layers between parallel and antiparallel orientations. A uniaxial anisotropy in both ferromagnetic layers was induced by depositing these layers in a magnetic field along the y-axis. The uniaxial anisotropy increased the squareness of the hysteresis loops and allowed the magnetizations to be antiparallel over a wide field range. Based on scanning electron microscope (SEM) images of the devices, like the image shown in Fig. 2, it was determined that $\theta=(30 \pm 5)^{\circ}$ giving $L=(42 \pm 2) \mathrm{nm}$. The $\mathrm{Cu}$ wires used as channels in these devices were deposited by electron beam evaporation and pattered by electron beam lithography to be $330 \mathrm{~nm}$ wide and $50 \mathrm{~nm}$ thick. The edges of the ferromagnetic layers were cleaned by ion milling immediately before depositing the $\mathrm{Cu}$ wires to remove any oxide. The resistance area product of the interface between the $\mathrm{Cu}$ channel and each of the FM layers is approximately $5 \times 10^{-3} \Omega \mu \mathrm{m}^{2}$, much less than for a tunnel junction.

Transport measurements were made with a lock-in amplifier using bias currents of approximately $0.5 \mathrm{~mA}$ at a frequency of $10 \mathrm{~Hz}$. The magnetic fields, used to change the magnetizations of the two ferromagnetic layers, were applied along the y-axis. Nonlocal resistance data taken from a FENL spin valve device are shown in Fig. 3, Here $I$ is driven from $\mathrm{A}$ to $\mathrm{B}$ and $V$ is measured from $\mathrm{C}$ to $\mathrm{D}$ (refer to Fig. 1) so that the CoFe acts as F1 and the NiFe acts as F2. The data show clear differences between $R_{N L}$ in the parallel and antiparallel magnetization states making $\Delta R$ easily measurable. The magnetic properties of seven nominally identical devices were quite similar, and the magnitude of $\Delta R$ at $291 \mathrm{~K}$ varied from $1.4 \mathrm{~m} \Omega$ to $2.60 \mathrm{~m} \Omega$.

For quantitative analysis, a comparison must first be made between the interface resistance $R_{i}$ and the characteristic resistances $R_{N} \equiv \rho_{N} \delta_{s} / A_{N}$ and $R_{F} \equiv \rho_{F} \delta_{F} / A_{F}$, where $\rho_{N}, \rho_{F}$ and $\delta_{s}, \delta_{F}$ are the resistivities and spin diffusion lengths of the nonmagnetic and ferromagnetic $(\mathrm{F})$ metals, $A_{N}$ is the cross-sectional area of the $N$ channel, and $A_{F}$ is the area of the interface between injector (or detector) and the channel ${ }^{14}$. In our devices, we measure $1.7 \Omega \approx R_{i}>R_{N}=0.8 \Omega$ and $R_{F} / R_{N} \approx 1$. These devices are not in a regime of negligible interface resistance and we use Johnson-Silsbee theory to relate $\Delta R$ to empirically measurable parameters through the expression:

$$
\Delta R=\frac{\eta^{2} \rho_{N} \delta_{s}}{A_{N}} e^{-L / \delta_{s}},
$$


where $\eta$ is the geometric mean of the polarizations of the two ferromagnetic interfaces 7,12 .

The geometry of FENL spin valves prevents the fabrication of devices with $L>\delta_{s}$, precluding the possibility of determining $\delta_{s}$ from fits of the form $\Delta R(L)$. A comparison to theory can be made using a value for $\delta_{s}$ found by other researchers studying $\mathrm{Cu}$ with similar resistivity. At room temperature, published values of $\delta_{s}$ are $400 \mathrm{~nm}$ in $\mathrm{Cu}$ with $\rho_{N}=2.3$ $\mu \Omega \mathrm{cm}^{15}$ and $500 \mathrm{~nm}$ in $\mathrm{Cu}$ with $\rho_{N}=2 \mu \Omega \mathrm{cm}^{16}$ and $5.5 \mu \Omega \mathrm{cm}^{17}$. The resistivity of the $\mathrm{Cu}$ in our devices was $\rho_{N}=3.0 \pm 0.2 \mu \Omega \mathrm{cm}$, similar to $\mathrm{Cu}$ used by these other researchers. Assuming a median value, $\delta_{s}=(450 \pm 50) \mathrm{nm}$, we find a range of values of $\eta$ from $\eta=(5.9 \pm 0.4) \%$ for the interfaces in the device with $\Delta R=(2.60 \pm 0.05) \mathrm{m} \Omega$ to $\eta=(4.3 \pm 0.4) \%$ for the interfaces in the device with $\Delta R=(1.4 \pm 0.1) \mathrm{m} \Omega$. These values of $\eta$ lay in the middle of the range of $\eta$, from $2 \% \frac{18}{\underline{1}}$ up to $12 \% \frac{19}{}$, published for other all-metal NLSVs with low (but non-negligible) resistance ohmic interfaces, at room temperature.

To further investigate the properties of our FENL spin valves we measured three devices at cryogenic temperatures [see Fig. 3(b)]. The ratio of $\Delta R$ at room temperature to that measured at $79 \mathrm{~K}$ varied between $52 \%$ and $58 \%$ for these three devices even though the room temperature value was different for each device. The ratio measured in our devices was significantly higher than ratios measured in other published studies of planar NLSVs with $\mathrm{Cu}$ channels, where the ratio was $38 \%$ for devices with $L=250 \mathrm{~nm}$ and $15 \%$ for devices with $L=1650 \mathrm{~nm}^{15}$. In our FENL spin valves, $L=42 \mathrm{~nm}$ is much shorter than $\delta_{s}$ at both room and cryogenic temperatures. Therefore, we have $e^{-L / \delta_{s}} \sim 1$ at both temperatures and the exponential term contributes little to the temperature dependence of $\Delta R$.

Another way way to characterize our FENL spin valves is to measure the baseline resistance $R_{B} \equiv\left(R_{N L}(\right.$ Parallel $)+R_{N L}($ Antiparallel $\left.)\right) / 2$. The baseline resistance depends on $L$, $\rho_{N}$, the width of the channel and the homogeneity of the interface resistances 20 . For our devices, theory ${ }^{20}$ predicts a range of possible values for $R_{B}$, between $0 \Omega$ if the interface resistances are homogeneous and $-0.1 \Omega$ if the interface resistances are characterized by point contacts on opposite sides of the channel. Measured values of $R_{B}$ for all seven devices were negative, as predicted by theory, but only one had a measured $R_{B}$ in the predicted range. The remaining devices had values between $-0.13 \Omega$ and $-7.35 \Omega$. The discrepancy between calculation and experiment is not understood, but may involve factors related to the unusual nonplanar geometry or the roughness of the edge (refer to Fig. 2). We note that while $R_{B}$ varied by two orders of magnitude in these seven devices the values of $\Delta R$ were all in the 
narrow range $2.0 \pm 0.6 \mathrm{~m} \Omega$.

There are several possible uses for FENL spin valves which take advantage of their unique properties. One promising possibility is to investigate spin momentum transfer effects ${ }^{2}$. The torque delivered by spin momentum transfer will act on a small volume of the FM material near the edge in these devices, possibly lowering the critical currents for switching and making them attractive for MRAM applications. Another use for these devices is to study the magnetization dynamics at the edge of ferromagnetic films, which are important to understand switching in small elements $\underline{21} 22$. FENL spin valves are uniquely suited to observe these edge modes because the nonlocal resistance of the devices is determined by the direction of the magnetization within a few nanometers of the edge.

More generally, our data clearly demonstrate that spin polarized current can be injected efficiently from the edge of a thin ferromagnetic film. The film thickness of our prototypes, $d_{F}=10 \mathrm{~nm}$, could presumably be made much smaller, $d_{F} \sim 1 \mathrm{~nm}$. This suggests interesting possibilities. A ferromagnetic layer could be fabricated in a trench, in a plane perpendicular to the surface of a chip. The film edge could be incorporated as part of a current perpendicular to the plane (CPP) spin valves. Such a film edge CPP spin valve would be expected to have the relatively large GMR of planar CPP spin valves with a lower resistance than MTJs. The cell area could be an order of magnitude smaller than that of either of these planar devices.

In conclusion we have introduced a new nonplanar geometry that uses the edge of a multilayer film, and adapted it to fabricate lateral nonlocal spin valves in which the distance between ferromagnetic electrodes is the order of ten nanometers. This enabled a study of spin accumulation in the unexplored size regime where the electrode spacing $L$ is much smaller than the spin diffusion length. The nonlocal resistance changes in these devices are consistent with Johnson-Silsbee theory. These results demonstrate that spin can be injected from the edge a film through narrow interfaces about 30 atoms wide. The relatively large resistance changes, small interface areas and the edge specific nature of their operation may make FENL spin valves useful for fundamental studies and applications.

Acknowledgement: The authors are grateful for the support of the Office of Naval Research, funding document N0001408WX20705, and they gratefully acknowledge use of the facilities at the NRL Nanoscience Institute. 
* Electronic address: mccallum@anvil.nrl.navy.mil

1 I. Zutic, J. Fabian and S. Das Sarma, Rev. Mod. Phys. 76, 323 (2004).

2 A. Mourachkine, O.V. Yazyev, C. Ducati and J.-Ph. Ansermet, Nano Lett. 8, 3683 (2008).

${ }^{3}$ M. Johnson and R. H. Silsbee, Phys. Rev. Lett. 55, 1790 (1985).

${ }^{4}$ M. Utrech, V. Korenivski, N. Poli and D. B. Haviland, Nano Lett. 6, 871 (2006).

${ }^{5}$ X. Lou, C. Adelmann, S. A. Crooker, E. S. Garlid, J. Zhang, K. S. M. Reddy, S. D. Flexner, C. J. Palmstrom, and P. A. Crowell, Nat. Phys. 3, 197 (2007).

${ }^{6}$ D. Saha, M. Holub, P. Bhattacharya, and Y. C. Liao, Appl. Phys. Lett. 89, 142504 (2006).

7 S. O. Valenzuela and M. Tinkham, Appl. Phys. Lett. 85, 5914 (2004).

8 Nikolaos Tombros, Csaba Jozsa, Mihaita Popinciuc, Harry T. Jonkman and Bart J. van Wees, Nature (London) 448, 571 (2007).

9 W. H. Butler, X.-G. Zhang, T. C. Schulthess, D. M. C. Nicholson, J. M. MacLaren, V. S. Speriosu, and B. A. Gurney, Phys. Rev. B 56, 14574 (1997).

10 M. D. Stiles, J. Appl. Phys. 79, 5805 (1996).

11 K. Xia, M. Zwierzycki, M. Talanana, P. J. Kelly, and G. E. W. Bauer, Phys. Rev. B 73, 064420 (2006).

12 M. Johnson, J. Appl. Phys. 75, 6714 (1994).

13 M. Heiblum, S. Wang, J. Whinnery, and T. Gustafson, IEEE J. Quantum Electron. 14, 159 (1978).

14 M. Johnson and R. H. Silsbee, Phys. Rev. B 35, 4959 (1987).

15 T. Kimura, T. Sato, and Y. Otani, Phys. Rev. Lett. 100, 066602 (2008). 
16 T. Kimura, J. Hamrle, and Y. Otani, Phys. Rev. B 72, 014461 (2005).

17 S. Garzon, I. Zutic, and R. A. Webb, Phys. Rev. Lett. 94, 176601 (2005).

18 A. Hoffmann, Phys. Status Solidi C, 4, 4236 (2007).

19 R. Godfrey and M. Johnson, Phys. Rev. Lett. 96, 136601 (2006).

20 M. Johnson and R. H. Silsbee, Phys. Rev. B 76, 153107 (2007).

21 J. Jorzick, S. O. Demokritov, B. Hillebrands, M. Bailleul, C. Fermon, K. Y. Guslienko, A. N. Slavin, D. V. Berkov, and N. L. Gorn, Phys. Rev. Lett. 88, 047204 (2002).

22 B. B. Maranville, R. D. McMichael, S. A. Kim, W. L. Johnson, C. A. Ross, and J. Y. Cheng, J. Appl. Phys. 99, 08C703 (2006). 


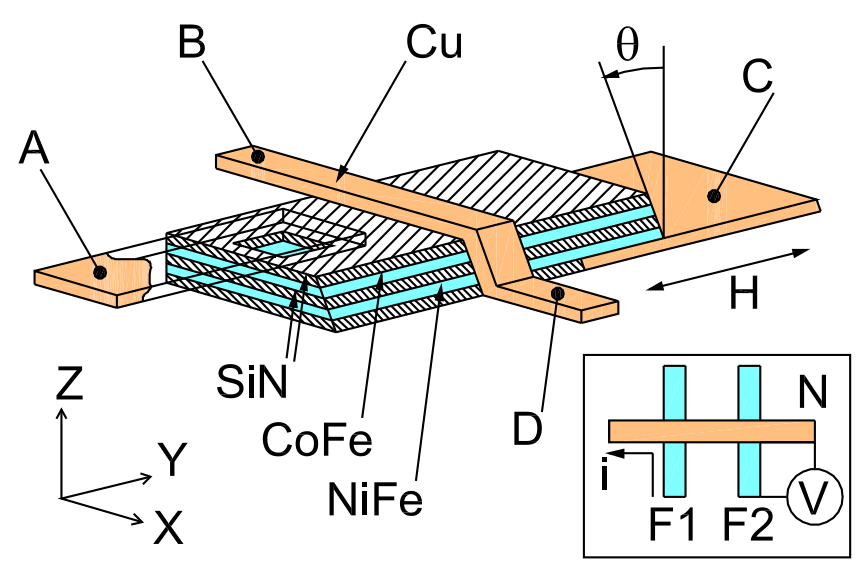

FIG. 1: (Color online) A perspective diagram of a FENL spin valve. The distance between the ferromagnetic contacts along the $\mathrm{Cu}$ channel is defined by the thickness of the insulator (SiN) separating the two layers. Nonlocal resistance measurements are made either by injecting current at contact $\mathrm{A}$, with a ground at $\mathrm{B}$, and measuring the voltage between contacts $\mathrm{C}$ and $\mathrm{D}$ or injecting current at contact $\mathrm{C}$, with a ground at $\mathrm{D}$, and measuring the voltage between contacts $\mathrm{A}$ and $\mathrm{B}$. Inset: Top view of a lateral spin valve. 


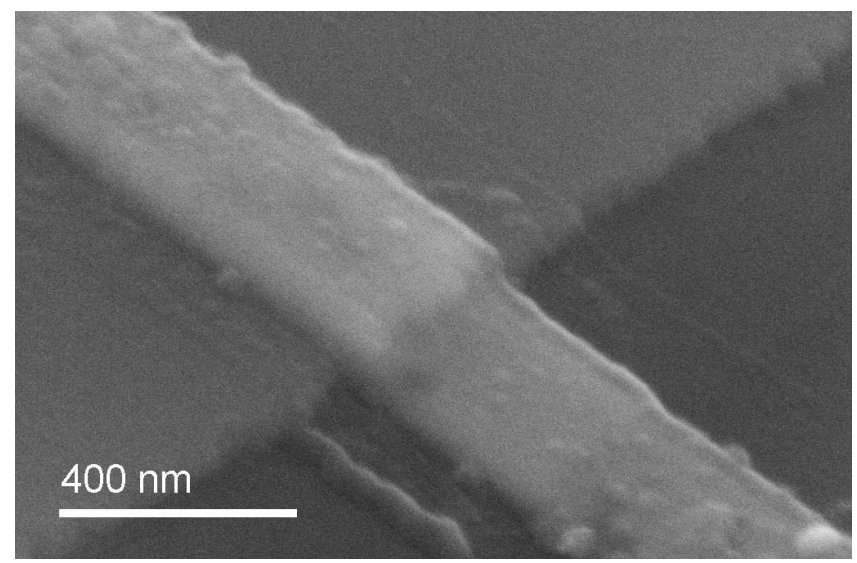

FIG. 2: An SEM image of a FENL spin valve taken at a 45 degree angle from the direction perpendicular to the sample plane. Note that the small thickness of the edge provides two independent and isolated electrical contacts to separate injector and detector films.

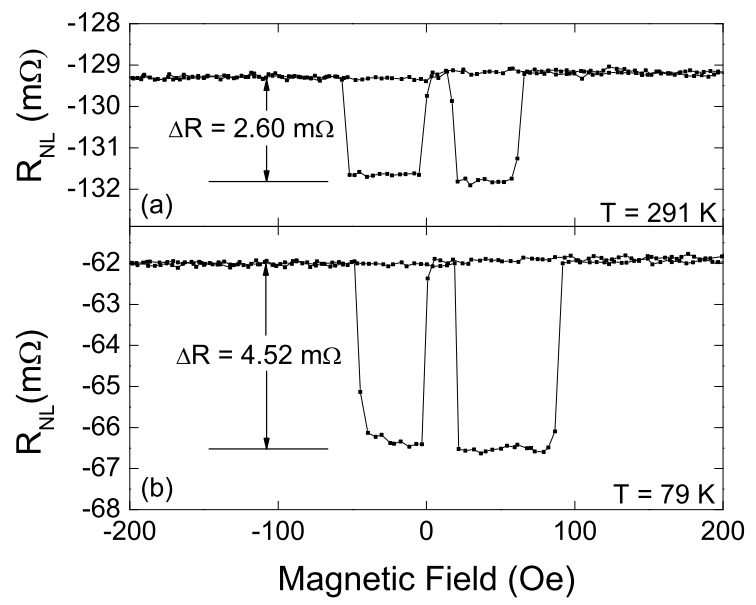

FIG. 3: Nonlocal resistance data from FENL spin valve device 15 on chip "B" as a function of applied magnetic field at $291 \mathrm{~K}(\mathrm{a})$ and $79 \mathrm{~K}(\mathrm{~b})$. 